'Departamento de Psiquiatría. División de Neurociencias. Facultad de Medicina. Pontificia Universidad Católica de Chile. Santiago, Chile. ${ }^{2}$ Centro de Bioética. Facultad de Medicina. Pontificia Universidad Católica de Chile. Santiago, Chile. aResidente del Programa de Psiquiatría del Adulto. Facultad de Medicina. Pontificia Universidad Católica de Chile.

Este trabajo no cuenta con fuentes de apoyo financiero de ningún tipo.

Recibido el 5 de octubre de 2015, aceptado el 13 de junio de 2016.

Correspondencia a: Sebastián Robert Barros Monroe 6974, Las Condes, Santiago. srobertbarros@gmail.com

\section{Evaluación de la capacidad del paciente para emitir un consentimiento válido: ¿es posible la estandarización?}

\author{
SEBASTIÁN ROBERT B. ${ }^{1, a}$, JUAN I. RECULÉ ${ }^{1, a}$, JUAN A. PRATO ${ }^{1,2}$
}

\section{Assessment of patients' capacity to give a valid consent}

Autonomy is an inherent condition of every ethical act. This attribution is expressed, when decisions are made, as capacity. Physician-patient alliance is defied when there is a reasonable doubt of patients' ability to make a choice, an extraordinarily complex scenario. As a response, in the last few decades multiple tools have been developed aiming to determine in a standardized fashion whether capacity is present or not. In the present article, we present a classification of the most well-known tools and discuss their usefulness, the implications for standardization of capacity, and make recommendations for their use, based in evidence.

(Rev Med Chile 2016; 144: 1336-1342)

Key words: Bioethics; Mental Competency; Personal Autonomy.
1 1 modelo de atención médica ha experimentado cambios progresivos durante los años, pasando de una dinámica asimétrica (paternalista), en la cual el médico toma decisiones en forma unilateral ante un paciente pasivo, a un modelo simétrico entre médico y paciente, en la cual el primero aporta con sus conocimientos y experiencia para poder tomar decisiones en conjunto con el segundo, asegurando la participación del paciente en el manejo de su propia salud.

Esta actual tendencia en el actuar médico, que pone particular énfasis en la autonomía del enfermo, reconociendo su enorme valor, está dotada de ventajas, principalmente para los pacientes. Sin embargo, no está exenta de dificultades para los médicos, quienes a pesar de su categoría de "aliados" del enfermo, no pueden escapar a las responsabilidades y obligaciones que su condición profesional de tratantes les confiere, ni ocultarse detrás de la autonomía del paciente para eludirlas al momento de tomar una decisión.

Muchas veces, esta contraposición genera paradojas para los profesionales enfrentando dos condiciones tan importantes como: a) la autodeterminación de las personas; y b) el principio de beneficencia y protección. En casos extremos, estas condiciones se vuelven irreconciliables entre ellas, de forma que no se puede respetar una sin desconocer la otra.

Cabe entonces hacerse las siguientes preguntas: ¿Es la autonomía del paciente lo más importante a la hora de tomar una decisión? ¿Cuándo es garantizable esta autonomía? Y finalmente pero más importante: ¿Cuándo existe verdadera autonomía?

Para formar alianza terapéutica (siempre basada en la autonomía del paciente y garante de su ejercicio), será un deber del médico reconocer si su estado limita su autonomía, expresada en lo que llamamos "capacidad".

El objetivo de este trabajo es dar una idea general sobre las herramientas descritas en la literatura para ayudar a los médicos a determinar la capacidad de los pacientes, las cuales, pese a existir desde hace casi tres décadas, rara vez son incorporadas a la práctica clínica en nuestro país. 


\section{Sobre el concepto de "autonomía", "competencia"y "capacidad"}

Dentro del marco de la Ética de Ley Natural, consideramos como actos libres todos aquellos actos realizados por personas sin coerción y en conocimiento de las alternativas. A aquellos actos libres que se orientan al fin último del ser humano, entendido como la consecución del bien y la autorrealización, los conocemos como actos éticos. Sólo éstos poseen la propiedad de la autonomía, o derecho absoluto a no ser interferidos por otras personas ${ }^{1}$. La autonomía se expresa en las figuras de lo que llamamos "capacidad" y "competencia", términos que pueden llevar a confusión por su similitud.

Con "competencia" nos referimos específicamente al reconocimiento legal de las aptitudes mentales necesarias para tomar decisiones. Por otro lado, "capacidad" es un término clínico, que hace referencia a las aptitudes mentales necesarias para tomar una decisión explícita en un momento y lugar determinados.

Pablo Simón-Lorda hace una sugerencia práctica al plantear reemplazar estos términos (muchas veces usados indistintamente, sobre todo en la literatura anglosajona), por los de "capacidad de derecho" refiriéndose a la competencia, y "capacidad natural o de hecho" refiriéndose a la capacidad propiamente tal ${ }^{2,3}$. El diálogo entre estos conceptos legal y ontológico de la cualidad del decidir y su conversación con las diferencias entre acto libre y acto ético escapan al propósito de este texto (la evaluación clínica del acto ético en ejercicio de su capacidad). Dicho tema es abordado por Bórquez y cols ${ }^{4}$.

Finalmente, aclaramos que al hablar de capacidad, es "capacidad para", y ese "para" es siempre un acto específico. No se es capaz o incapaz en general, sino siempre circunscrito a una tarea definida.

\section{Sobre los dominios de la capacidad}

Una vez definida la capacidad como aquellas aptitudes mentales necesarias para tomar una decisión definida en un momento y lugar específicos, surge la pregunta obvia: ¿Cuáles son estas aptitudes? Escapa a los objetivos de este trabajo detallar el proceso que llevó a la determinación de estos conceptos esenciales y nos limitaremos a mencionarlos y describirlos. En general, las herramientas que se han creado para evaluar la capacidad de los pacientes se relacionan con alguno de estos dominios, o todos ellos:

\section{1) Comprensión (understanding)}

Hay consenso en la definición de este concepto. Consiste en la habilidad del paciente de reconocer información relevante sobre los diagnósticos y eventuales tratamientos que se le plantean. Existe comprensión cuando el paciente puede repetir o parafrasear la información entregada por el médico 5 .

\section{2) Apreciación (appreciation)}

Es el uso de la comprensión para relacionar la información recibida con una situación en particular, unas circunstancias determinadas $y$ un entorno de creencias específico ${ }^{6,7}$. A diferencia de la comprensión, no existe un consenso muy claro en cuanto a qué se entiende exactamente por apreciación, y no se ha logrado correlacionar de forma satisfactoria este ítem en particular al comparar las herramientas específicas para evaluar capacidad con otros tests, como son las pruebas neuropsicológicas. Esto puede deberse a que la apreciación es un concepto más abstracto.

La forma específica para evaluar este dominio varía de una herramienta a otra, pudiendo basarse en las expectativas del paciente sobre los tratamientos, sus sospechas o reparos, o la expresión de dudas razonables.

\section{3) Razonamiento (reasoning)}

Consiste en la habilidad de imponer la lógica y la racionalidad en la evaluación de las alternativas en una situación determinada, para tomar la "mejor decisión”. Esta capacidad puede reconocerse en los pacientes cuando identifican y comparan los riesgos y beneficios, defienden sus decisiones de forma lógica, presentan un buen nivel de insight y juicio o cuando se reconocen los valores subyacentes que influencian la toma de una decisión (esto último es particularmente importante cuando la decisión no parece lógica a ojos del médico en primera instancia).

\section{4) Expresión de una decisión (expressing a choice)}

Probablemente la más simple de evaluar, consiste en la habilidad del paciente para comunicar 
una preferencia. El consenso en esta definición es amplio ${ }^{6}$.

Estas dimensiones son progresivas. La separación es, por supuesto, académica, puesto que todas están naturalmente hilvanadas en la continuidad del tomar la decisión.

\section{Sobre las herramientas para evaluar capacidad}

Ya en 1977, Roitz, Meisel y Lidz reconocían estos criterios centrales para determinar la capacidad $^{8}$, y desde entonces se han desarrollado múltiples herramientas destinadas a evaluarlas, intentando crear un modelo estandarizado y reproducible. Existen diferentes formas de clasificar estas herramientas. Simón-Lorda nos presenta una particularmente sencilla ${ }^{2}$.

\section{Tests neuropsicológicos}

De ellos, los más conocidos y utilizados son el Mini Mental State Examination (MMSE) y la Escala de Inteligencia de Weschler (WAIS). A pesar de que no corresponden a herramientas diseñadas específicamente para la evaluar capacidad, resultan útiles como apoyo a otros tests, sobre todo considerando su validez.

Se ha demostrado buena correlación entre el poder de predicción de estas herramientas y otros scores específicos que mencionaremos más adelante, sobre todo en relación a la comprensión y razonamiento. No ocurre lo mismo al momento de evaluar apreciación ${ }^{9}$. Se ha sugerido ya que este tipo de herramientas enfocadas en la evaluación de la atención, el lenguaje, la memoria o la función ejecutiva deberían incorporarse en la evaluación de la capacidad habitualmente ${ }^{10}$.

\section{Protocolos específicos de evaluación de capacidad}

Corresponden a aquellas pruebas diseñadas para evaluar la capacidad del paciente de consentir o rechazar en relación a decisiones específicas. Un buen ejemplo es el "Competence Interview Schedule" o CIS por sus siglas en inglés, protocolo destinado a evaluar la capacidad de aceptar o rechazar la terapia electroconvulsiva ${ }^{11}$. Este tipo de herramientas es bastante útil, sin embargo, su uso es acotado, por lo que no resultan idóneas para la evaluación por el médico general.

\section{Protocolos generales de evaluación de capacidad}

Son herramientas para evaluar capacidad, aplicables a cualquier tipo de situación. La mayoría evalúa los cuatro dominios de capacidad a la vez, y pueden, a su vez, clasificarse en:

a. Guiones de entrevistas: más que una verdadera herramienta, corresponden a un conjunto de preguntas sugeridas a los clínicos para realizar una exploración básica de la capacidad. No tienen instrucciones específicas sobre su aplicación. Probablemente una de las más conocidas sea la lista de preguntas sugeridos por Moye ${ }^{10}$.

b. Protocolos estructurados o semi estructurados: Son las pruebas más complejas, incluyendo algunas de las más conocidas y validadas como el "MacArthur Competence Assessment Tool for Treatment" (MacCAT-T) ${ }^{12}$. Estos guiones suelen estar asociados a un sistema de evaluación de respuestas expresado en un puntaje; no se plantean como scores dicotómicos para responder si el paciente es o no capaz, sino más bien como un continuo desde la nula a la completa capacidad, visión que traduce la realidad clínica.

c. Protocolos basados en viñetas: Estos consisten en descripciones de situaciones imaginarias sobre las cuales el paciente debe tomar decisiones relacionadas a la salud. Los estudios en relación a estas pruebas son escasos, $y$ han demostrado mala correlación con otras herramientas ${ }^{13}$, aunque podrían ser útiles en grupos específicos como los adultos mayores con demencia ${ }^{14}$.

La Tabla 1 menciona algunas de las principales herramientas usadas para la evaluación de la capacidad.

\section{Sobre la validación e implementación de las escalas}

La elección de la herramienta apropiada dependerá de múltiples factores. Primeramente, existen escalas destinadas a ciertos grupos de pacientes (como los métodos de viñetas, orientadas a adultos mayores con o sin diagnóstico de demencia) o a ciertos tipos de decisión (como los protocolos específicos). Además, el tiempo que requieren para su implementación es también variable. Así, es poco probable que pruebas demasiado largas puedan usarse en todos los casos. 
Tabla 1. Principales herramientas usadas para la evaluación de capacidad

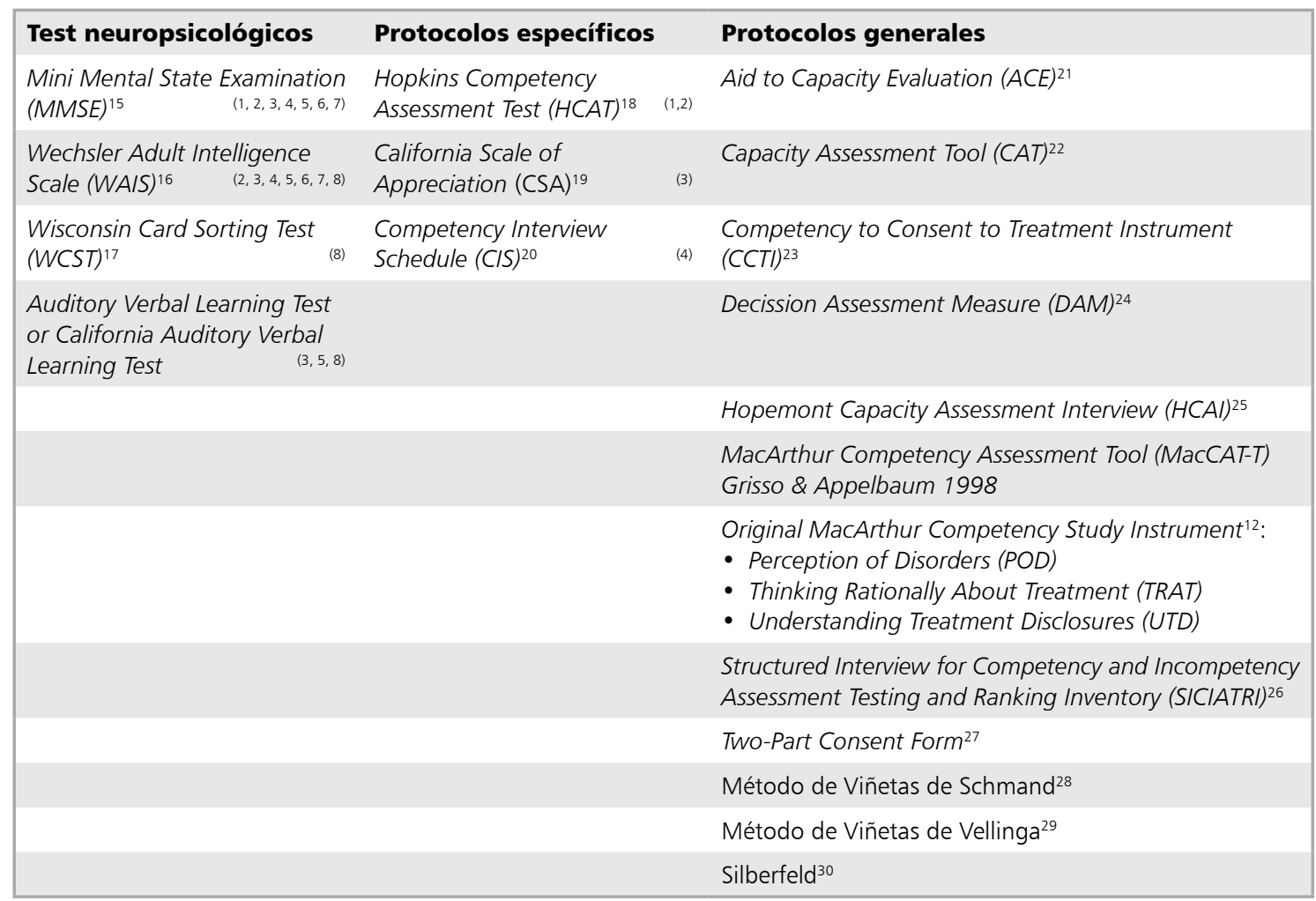

Las dimensiones neurocognitivas y de la competencia evaluadas por cada test neuropsicológico de la primera columna están señaladas por superíndice y corresponden a: 1 Orientación espacio temporal, 2 Capacidad de atención, 3 memoria, 4 Capacidad de abstracción (cálculo), 5 Capacidad de lenguaje, 6 percepción viso-espacial, 7 Comprensión, 8 Función ejecutiva. Para los protocolos específicos, los contextos específicos de uso para cada escala o test están señalados por superíndices y corresponden a: 1 Consentimiento informado; 2 Escritura de instrucciones anticipadas; 3 Consentimiento para investigación; 4 Aceptación o rechazo de terapia electroconvulsiva.

Será siempre preferible para el uso de médicos generales herramientas que requieran poca capacitación, como es el caso del "Hopkins Competency Assessment Test"(HCAT) y que consuman poco tiempo, como el MacCAT-T, que puede realizarse en aproximadamente 10 a $15 \mathrm{~min}$.

Adicionalmente, los procesos de validación de estas pruebas son difíciles. La evidencia es escasa ${ }^{7}$, pues muchos de estos instrumentos no cuentan con segundos estudios que los avalen, carecen de consistencia interna probada ${ }^{31}$ o no han demostrado ser reproducibles de forma satisfactoria entre distintos entrevistadores ${ }^{7,32}$.

Es interesante mencionar a su vez que no existen muchos estudios que comparen estas herramientas entre sí. Una comparación realizada entre el MacCAT-T, el CCTI y el HCAI demostró un acuerdo moderado al evaluar la capacidad en general, sin embargo, al desglosar según los dominios específicos, se observó que mientras el grado de acuerdo en relación a la comprensión era bueno, no lo era para razonamiento ni apreciación (en este último caso no es mejor que el azar) ${ }^{33}$.

Por último, la falta de un gold standard apropiado hace difícil una comparación entre los métodos. En numerosas ocasiones se ha pretendido comparar la predicción de capacidad con las entrevistas realizadas por psiquiatras expertos o médicos generales. Se pueden destacar en estos casos el test de Silberfeld, en cuanto a su buena correlación con la evaluación por psiquiatras ${ }^{34} \mathrm{y}$ el HCAT por su mejor correlación con las evaluaciones por médicos generales ${ }^{35}$. Sin embargo, son legítimas las dudas sobre qué parámetros y 
condiciones debe cumplir un especialista para ser considerado "experto". A la fecha es poco el grado de consenso en estas definiciones.

\section{Sobre la incorporación de herramientas a la práctica clínica habitual}

La elección de una herramienta universal definitiva de evaluación de la capacidad para la clínica es impracticable. Lo cierto es que dependiendo del caso particular algunos scores podrían ser más útiles que otros. Sin embargo, si reunimos la evidencia disponible en las últimas dos décadas, una buena primera herramienta debiera cumplir los siguientes estándares:

1. Que evalúe los cuatro dominios a la vez. Este criterio ya permite seleccionar un número limitado de herramientas, a saber: ACCT, ACT, CAT, CCTI, DAM, MacCAT-T, Mac Arthur Original, Silberfeld, SICIATRI, Método de viñetas de Schmand, Método de viñetas de Vellinga $^{7-9}$.

2. Que haya probado consistencia inter operador: A su vez nos permite descartar entre los ya mencionados los métodos de viñetas de Schmand y Vellinga ${ }^{8,10,11}$.

3. Que haya probado tener consistencia interna: La evidencia en este aspecto sólo respalda a las escalas MacCAT-T y Mac Arthur Original ${ }^{6,31,32}$.

4. Que requiera poca capacitación y breve tiempo para aplicarse: En este sentido el MacCAT-T es superior ${ }^{6,7}$.

Proponemos, entonces, como herramienta de primera línea el MacCAT-T que, además de ser comprehensiva en su evaluación de los dominios de la capacidad, ha demostrado un buen grado de consistencia interna, reproducibilidad entre distintos entrevistadores y múltiples evaluaciones en un mismo paciente, y que puede aplicarse en poblaciones diversas, con o sin antecedentes de patología psiquiátrica. Además, como ya dijimos, puede implementarse en un tiempo relativamente corto y no requiere mayor entrenamiento. La Tabla 2 ilustra el contenido de esta herramienta ${ }^{2}$.

La necesidad de evaluar formalmente capacidad es más urgente cuanto más dudosa es esta. Si bien el gold standard sigue siendo la evaluación por el clínico experimentado, una herramienta permite documentar y sistematizar dicha evaluación. Sugerimos la incorporación del MacCAT-T como primera herramienta de apoyo a la entrevista clínica que todo médico debe realizar en caso de que existan dudas legítimas en materia de capacidad.

No se debe olvidar que responder estas dudas es un deber del médico, y sobre todo, que poner en tela de juicio la capacidad de un paciente para tomar decisiones (cuando parece sensato) no es caer en una actitud paternalista, sino muy por el contrario, es demostrar compromiso y responsabilidad ante el paciente, para así lograr una alianza terapéutica eficaz que asegure la verdadera autonomía.

\section{Conclusiones}

La medición de la capacidad es un proceso complejo, que depende de múltiples variables, y que puede representar un desafío incluso para

Tabla 2. Criterios y puntuaciones del MacArthur Competency Assessment Tool (MacCAT-T)

\begin{tabular}{|llcc|}
\hline Criterio & Subcriterio & Puntaje parcial & Puntaje total \\
\hline Comprensión & Comprensión de la enfermedad & $0-2$ \\
& Comprensión del tratamiento & $0-2$ & $0-2$ \\
Apreciación & Comprensión de los riesgos y beneficios & $0-2$ & $0-6$ \\
& De la situación de la enfermedad & $0-2$ & $0-4$ \\
\hline Razonamiento & Del objetivo general del tratamiento & $0-2$ & \\
& Lógico-secuencial & $0-2$ & $0-2$ \\
& Lógico-comparativo & $0-2$ & $0-8$ \\
\hline \multirow{2}{*}{ Elección } & Predictivo (consecuencias derivadas de las posibles elecciones & $0-2$ & $0-2$ \\
\hline
\end{tabular}


médicos especialistas en la materia. Por esta razón se han creado múltiples herramientas destinadas a facilitar esta evaluación. Sin embargo, no existe una herramienta que sea perfecta, pues todas ellas tienen limitaciones y ventajas.

El consenso en relación a qué se debe medir aparentemente existe, pero muchas de las definiciones respecto a los dominios de capacidad a evaluar aún no están estandarizadas.

Hasta la fecha, el MacArthur Competency Assessment Tool (MacCAT-T) aparece como la herramienta más validada. Destacamos que no es posible una evaluación dicotómica sobre si un paciente está o no capacitado para tomar una decisión, por ello este test, como los demás presentados, evalúan en forma cuantitativa la capacidad como un espectro, siendo un apoyo (pero no un reemplazo) para el juicio clínico a la hora de tomar una determinación.

Sugerimos la incorporación de la escala MacCAT-T como una herramienta que debiera estar disponible y ser conocida por todos los clínicos, que podrían encontrar en ella un argumento adicional y en ocasiones de altísimo valor, para tomar decisiones responsables en esta delicada y compleja materia.

\section{Referencias}

1. Reculé JI. Autonomía en el Paciente Psiquiátrico. Rev GPU 2014; 10 (1): 108-15.

2. Simón-Lorda P. La capacidad de los pacientes para tomar decisiones: una tarea todavía pendiente. Rev Asoc Esp Neuropsiq 2008; 28 (102): 325-48.

3. Sullivan K. Neuropsychological Assessment of Mental Capacity. Neuropsychology Review 2004; 14 (3): 131-42.

4. Bórquez G. La evaluación de la capacidad de la persona: en la práctica actual y en el contexto del consentimiento informado. Rev Med Chile 2004; 132: 1243-8.

5. Lamont S. Assessing patient capacity to consent to treatment: an integrative review of instruments and tools; Jour of Clin Nur 2013; 22: 2387-403.

6. Sturman ED. The capacity to consent to treatment and research: a review of standardized assessment tools. Clin Psychol Rev 2005; 25: 954-74.

7. Moye J, Karel MJ, Edelstein B, Hicken B, Armesto JC, Gurrera RJ. Assessment of capacity to consent to treatment: challenges, the "ACCT" approach, future directions. Clin Gerontol 2008; 31: 37-66.

8. Roth LH, Meisel A, Lidz CW. Tests of Competency to
Consent to Treatment. Am J Psychiatry 1977; 134 (3): 279-84.

9. Moye J, Marson DC. Assessmentof decision-making capacity in older adults: an emerging area of practice and research. J Gerontol B Psychol Sci Soc Sci 2007; 62 (1): P3-P11.

10. Moye J. Assessment of competency and decisión making capacity: Lichtenberg, P. A. (ed.), Handbook of Assessment in Clinical Gerontology (pp. 488-528), Wiley, New York. 1999.

11. Bean G, Nishisato S, Rector NA, Glancy G. Thepsychometric properties of the Competency Interview Schedule. Can J Psychiatry 1994; 39 (8): 368-76.

12. Grisso T, Appelbaum PS. Assessing Competence to Consent to Treatment: a Guide for Physicians and Other Health Professionals. Oxford University Press, New York 1998.

13. Marson DC, Hawkins L, McInturff B, Harrell LE. Cognitive models that predict physician judgments of capacity to consent in mild Alzheimer's disease. J Am Geriatr 1997; Soc 45: 458-64.

14. Schumand B, Gouwenberg B, Smitt JH, Jonker C. Assessment of mental competency in community-dwelling elderly. Alzheimer Dis Assoc Disord 1999; 13 (2): 80-97.

15. Folstein MF, Folstein SE, McHugh PR. "Mini-mental state". A practical method for grading the cognitive state of patients for the clinician. J Psychiatr Res 1975; 12 (3): 189-98.

16. Wechsler D. Manual for the Wechsler Adult Intelligence Scale. Psychological Corp. Oxford, England. 1955.

17. Grant DA, Berg E. A behavioral analysis of degree of reinforcement and ease of shifting to new responses in a Weigl-type card-sorting problem. J Exp Psychol 1948; 38 (4): 404.

18. Janofsky JS, McCarthy JS, Folstein MF. The Hopkins Competency Assessment Test: a brief method for evaluating patients' capacity to give informed consent. Hosp Community Psychiatry 1999; 43 (2): 132-6.

19. Saks ER, Dunn LB, Marshall BJ, Nayak GV, Golshan S, Jeste DV. The California Scale of Appreciation: a new instrument to measure the appreciation component of capacity to consent to research. Am J Geriatr Psychiatry 2002; 10 (2): 166-74.

20. Bean G, Nishisato S, Rector NA, Glancy G. The assessment of competence to make a treatment decision: an empirical approach. Can J Psychiatry 1996; 41 (2): 8592.

21. Etchells E, Darzins P, Silberfeld M, Singer PA, McKenny J, Naglie G, et al. Assessment of patient capacity to consent to treatment. J Gen Intern Med 1999; 14: 27-34.

22. Carney MT, Neugroschl J, Morrison RS, Marin D, Siu 
AL. The development and piloting of a capacity assessment tool. J Clin Ethics 2001; 12: 17-23.

23. Marson DC, Ingram KK, Cody HA, Harrell LE. Assessing the competency of patients with Alzheimer's disease under different legal standards. A prototype instrument. Arch Neurol 1995; 5: 949-54.

24. Wong JG, Clare CH, Holland AJ, Watson PC, Gunn M. The capacity of people with a 'mental disability' to make a health care decision. Psychol Med 2000; 30: 295-306.

25. Edelstein B. Hopemont Capacity Assessment Interview Manual and Scoring Guide. West Virginia University, Morgantown, WV 1999.

26. Tomoda A, Yasumiya R, Sumiyama T, Tsukada K, Hayakawa T, Matsubara, et al. Validity and reliability of structured interview for competency incompetency assessment testing and ranking inventory. J Clin Psychol 1997; 53: 443-50.

27. Roth LH, Lidz CW, Meisel A, Soloff PH, Kaufman K, Spiker DG, et al. Competency to decide about treatment or research: an overview of some empirical data. Int J Law Psychiatry 1982; 5: 29- 50.

28. Schmand B, Gouwenberg B, Smit J, Jonker C. Assessment of mental competency in community-dwelling elderly. Alzheimer Dis Assoc Disord 1999; 13: 80-7.

29. Vellinga A, Smit J, Van Leeuwen E, van Tilburg W,
Jonker C. Competence to consent to treatment of geriatric patients: judgements of physicians, family members, and the vignette method. Int J Geriatr Psychiatry 2004; 19: 645-54.

30. Fazel S, Hope T, Jacoby R. Assessment of competence to complete advance directives: validation of a patient centred approach. BMJ 1999; 318: 493-7.

31. Dunn LB, Nowrangi MA, Palmer BW, Jeste DV, Saks ER. Assessing decisional capacity for clinical research or treatment: a review of instruments. Am J Psychiatry 2006; 163: 1323-34.

32. Raymont V, Buchanan A, David AS, Hayward P, Wessely S, Hotopf M. The inter-rater reliability of mental capacity assessments. Int J Law Psychiatry 2007; 30: 112-7.

33. Gurrera RJ, Karel MJ, Azar AR, Moye J. Agreement between instruments for rating treatment decisional capacity. Am J Geriatr Psychiatry 2007; 15: 168-73.

34. Fassassi S, Bianchi Y, Stiefel F, Waeber G Assessment of the capacity to consent to treatment in patients admitted to acute medical wards. BMC Med Ethics 2009; 10: 15.

35. Wilkins KM, Lund BC, McAdams JD, Yates WR. Clinical utility of the Hopkins competency assessment test on an inpatient geropsychiatry unit. Am J Alzheimers Dis Other Demen 2009; 24: 34-9. 